\title{
NATURE AND IMPORTANCE OF THE PRIORITIES IN THE DEVELOPMENT PLANS
}

\author{
K. Krumov* \\ Department of Marketing and Strategic Planning, University of National and World Economy, \\ Sofia, Bulgaria
}

\begin{abstract}
The objective of the author with this work is to present the place and role of priorities in the development plans. The main reason for the consideration of this thematic is the existing practice of deduction and formulation of the priorities in the development plans in Bulgaria on all levels. The wrong approaches and low quality of the priorities in these plans create serious difficulties at a moment when the country as a whole, all territorial and economic units in it are in pressing need for development, growth and increase of the incomes. The incorrectly set priorities strongly affect the quality of the plans and options for the realization of these plans. This is the reason for the author to present through the prism of the system approach several key directions for consideration of the priorities and thus to contribute to the change of the existing practice.
\end{abstract}

Key words: priorities, development plans

\section{Essence of the priorities}

The system approach on the essence and meaning of the priorities in the development plans require their study from several view points. A starting moment in this process is the definition for priority. According to the general theory and elementary logic, ,a priority" is something more urgent and important than all the rest. In other words, this „,something” must be performed or realized before all the rest and/or is of bigger importance or rang in the whole it is a part of.

Based on this definition, the deduction and place of the priorities in the plan has been considered in several key directions for their nature.

\section{First - specificity of the field}

The objects in each field of the human activity have their own specificity against the objects of the other fields. According to this specificity, consideration should be given to the fact that the development plans on different levels (supranational, national, regional, branch and company level) directly affect the social economic and technological systems, i.e. affect several fields simultaneously. This gives

\footnotetext{
${ }^{*}$ Correspondence to: Chief assistant professor Kalin Krumov, PhD, Sofia, Bulgaria Address: 1700 Sofia, Students Town, UNWE, E-mail: krumov.unwe@gmail.com, Phone: 0878945927
}

us the ground to make a correction and to indicate that the priorities in the development plans are the most urgent and important social economic and technological needs against all the other needs in these fields. The presence of several fields requires the investigation of their interaction, connections and dependences and on that ground, to bring forward the priorities to the development of the subject of planning. If the various fields are reviewed separately, the presence of own specificity in each will impose different priorities against the priorities that will be brought forward when the fields are reviewed in interaction. In this case it is likely that the priorities from different fields contradict to each other, as per the case, when reviewed as a whole. For instance the priorities in the social field contradicting the priorities in the economic field, the priorities in the economic field contradicting to the priorities in the field of ecology, etc. The partial approach to the development of an object presupposes the occurrence of such contradictions between the priorities.

Second - the priorities and system essence of the object of planning

Despite of the fact that the objects of planning consist of elements of various fields, they represent a part of a whole - national economy, separate territorial units, branches, companies. Each of these objects is by itself a 
system consisting of elements among which two-directional and multi-directional complex relations and interactions exist. In view of the interaction of the elements, it is an essential specificity that via the relations, the state (potential) of an element is transformed into a state potential of the rest of the elements and vice versa.

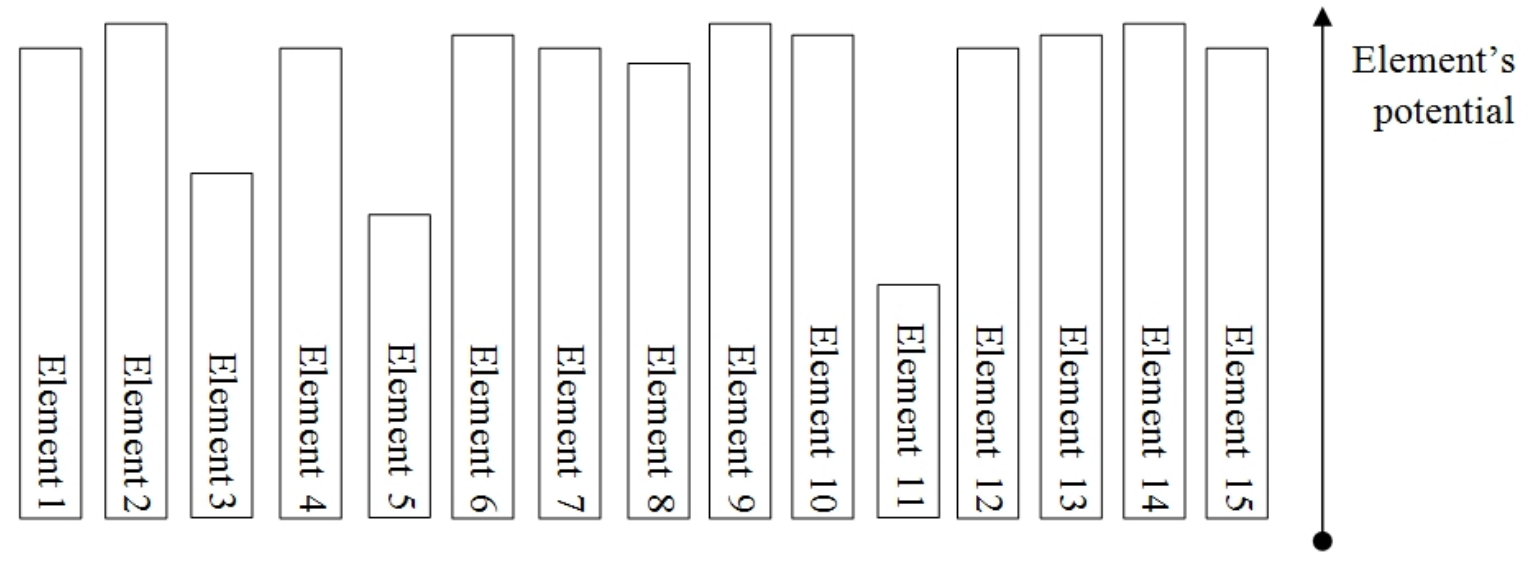

Figure 1.

Resource: own making.

This specificity is of essential importance in the deduction of priorities in the development plans of the objects, because if the potential of an element or a group of element is reduced, via the relations, this will result in the reducing of the potential of the rest of the elements and the system. I.e. the resultant of the interaction between the elements in the system is determined by the element of lowest potential. Figure № 1 presents a sample system of 15 elements, of which 3 have the lowest potential. If the non-compliance between the potentials of these elements with the rest of them is not overcome, these will limit the options for development in the future.

From this point of view, the process of deduction of priorities in the first place requires to give an answer to the following question: the development of which elements in the planning object could be priorities and why? To answer this question, an analysis is needed of the external and internal environment of the object. This analysis is to reveal:

- The favorable options and threats before the development of the object hidden in its external environment;

- Which elements or groups of elements in the object of planning are affected by the assimilation of the options or neutralization of the external threats;

- What is the state and potential of these elements or groups of elements of the system and to what extent does this state and this potential need to develop to enable the system to assimilate the external options and to neutralize the external threats.
- The state or potential of which elements or groups of elements prevent or limit the development of the system as a whole.

On that ground, the elements or groups of elements can be determined as critical for the system development, as follows:

- Represent an essential problem to the system development - their significantly lower potential against the potential of the rest of the elements of the system limits the development;

- Elements, for which the internal or external conditions reveal big opportunities for development which on its hand will stimulate the system development as a whole. An example in this respect can be the development of separate branches in a territorial unit which has specific natural resources - soils for development of the agriculture, useful ores for the industry, etc., or branches, the production of which has large variety of options for the realization of the external markets;

- Elements which have specific meaning and role for the system development as a whole. An example for that is the Education branch, the development of which is a prerequisite for the creation of human resource of high potential, these, on their side are the prerequisites for the creation of new knowledge, the new knowledge, introduced in the production are the prerequisite for the increase of the competitiveness of the economic and incomes, and so forth.

So far it is indicated that a theory of the systems can be used as a criterion for the determination of the development critical elements or groups of elements in a system 
which represent a priority for the system development in the future.

Third - elements and priorities in the plan critical for the development

This part reviews the connection between the elements of a system and the priorities in its development plan. It is extremely important to indicate that the elements of a system in quantitative and qualitative sense are distinct from the priorities in the plan - the first are a part of the planning object and the second ones are a part of the development plan of this object. The connection between them is expressed in the fact that the development of the elements or groups of elements including the ones critical for the development and representing a priority, is realized via the bringing forward of objectives for their development. In the plans for development on national, regional, branch and company level, priorities can be only separate objectives of the tree of objectives in the plan and not the resources, measures and actions to achieve these objectives. The logic presumes that the vision and main objective cannot be priorities because they have a specific role - the vision displays the future image and direction of development in the long term and cannot be achieved in the period the plan is developed for, whereas the main objective reveals the objective of the system during the plan period but cannot entirely achieve it. Besides, the difference between the main objective and subobjectives is expressed in the fact that via the main objective, the desired future state of the territorial unit is identified, and via the subobjectives - the desired future state of the separate subsystems or elements. Thus, in the ultimate, the priorities in the development plans are sub-objectives that have a specific status against the remaining sub-objectives of the plan.

Here occurs the question if priorities in the plans can be the resources for the achievement of certain sub-objective or the measures and actions brought forward for that? The answer is negative. The reason is that, although the measures, actions and resources are straightly directed to the achievement of the subobjectives, these do not display the future state and potential that the elements are desired to have at the end of the plan period. The through given demonstrate that priorities in the plans can only be the sub-objectives for the development of an element or a group of elements.

Forth - regarding the higher importance of the priorities in time

The priorities in view of time as a specific resource engender an interesting question: should the priorities by all means be realized before the rest of the sub-objectives of the plan structure? The answer negative or at least it is not all priorities. The reason for that is hidden in the non-contradictive decomposing of the main objective of the plan into sub-objectives, i.e. the decomposition is performed so as the sub-objectives of $3^{\text {rd }}$ rang result in the achievement of the sub-objectives of the $2^{\text {nd }}$ rang, and their achievement results in the achievement of the sub-objectives of 1-st rang, on its turn, the realization of the sub-objectives of 1-st rang results in the realization of the main objective of the plan. Practically the decomposition of this type defines the succession of realization of the sub-objectives. An important principle in the planning is that the objectives in the plans are deduced updown and are realized down-up - Figure № 2.

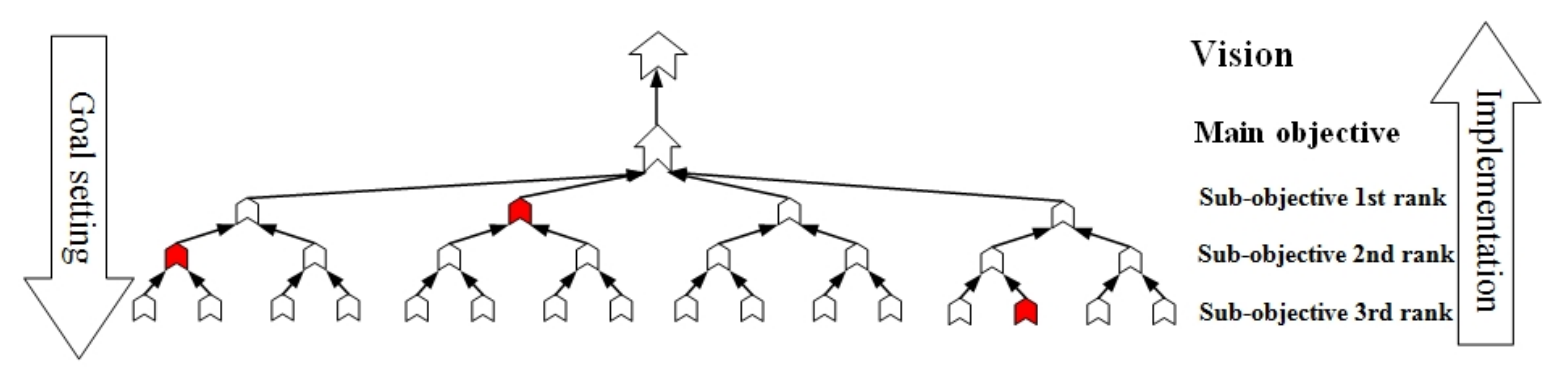

Figure 2. Goal and objective Structure.

\section{Resource: own making.}

Whether the priorities will be realized before the rest of the sub-objectives depends on their pace in this structure. On its turn, the place of the priorities in the structure of the objectives depends on the state and potential of the elements of the system (Figure № 1) - the priority elements are the ones of lowest potential or the ones whose development hides big opportunities for the system as a whole. Besides, the structure of the plan objectives (objective decomposing) is not random but follows a strict succession which is coordinated to the nature of the planning subject in its quality of a system, i.e. considers the elements of a system and interaction between them as well as the interaction of the system with the external environment. 
Thus the succession of realization of the priorities in time can be comprehended using Figure № 2. The realization of the objectives down-upward means first the realization of the sub-objectives of 3-rd rang including the first priority (marked red), then realization of the sub-objectives of the 2-nd rang and the second priority and at the end realization of the subobjectives of first rang among which is the last third priority.

Fifth - relation between priorities and resources in the plan

This concerns the matter whether for the realization of the sub-objectives, which are priority, more resources are spared against the rest sub-objectives in the plan? The answer to this question is positive - to satisfy the most important needs as are the priorities, more resources are spared. An explanation for this can be found using Figure № 1, which demonstrated variations in the potentials and elements of a system. On this Figure, elements 3,5 and 11 have a significantly lower potential. It was already indicated that if these differences will not be overcome, i.e. the potentials of these three elements are not approximately equalized with the potentials of the remaining ones (synchronized), elements 3 , 5 and 11 will impede the development. This is the main reason for the priorities which, in this case foresee that the development of elements 3, 5 and 11 are spared more resources to enable the equalization of the potential of these elements in future with the potential of the rest of the elements.

What is indicated here means that the priorities play the role of a criterion on the base of which the limited resources are distributed among the plan sub-objectives. With their help, a concentration is realized of financial, human material and other resources for the development of ,the critical points" of a system - the elements of the lowest potential or elements that can stimulate the development of the whole.

On the other hand, however, the distribution of more resources to the priorities can be relative in absolute value. The reason for that is in the nature and scales of the system elements, for which the priorities are deduced. For instance a sub-objective in the plan that is directed to the development of the technical infrastructure, more resources can be foreseen (financial, material, human and others), than for a subobjective that is a priority and is directed to the environmental preservation. In this case in absolute volume, most likely the resources directed to the development of the infrastructure will be twice as much as for the environmental preservation. Example for that can be some cities in China in which the air pollution by burnt gases is so strong that it is brought forward as a priority before the city development. Nevertheless, the resources for the development of the city infrastructure roads and communications in absolute size are more than these spared for the environment preservation. From this point of view, the concentration of resources in the priorities concerning the critical elements for the system development means sparing more resources in absolute value than would be spared for the same element when in normal state or having similar potential and importance with the rest elements of the system. In the practice, both can happen - the priorities could concentrate more resources in absolute or relative terms.

Sixth - the priorities in the various plan periods

On the next place, it is important to pay attention to one more question - is it possible that, during the different plan periods, the same sub-objectives or similarly formulated subobjectives to be priorities. Generally, during the various plan periods, the various subobjectives can play the role of priority however this does not mean that the same sub-objective cannot be a priority in several consecutive plan periods. As a whole, if in a plan for development of an object, one of the priorities envisages the increase of the competitiveness of the economic to certain level, this does not mean that in the plan for the next period such priority cannot exist. Which sub-objective will be a priority depends only on the state of the object of planning and its compounds as well as the nature of their interaction with their external environment. If a system element has a very low potential, the process of increase of this potential will most likely be continuous and concern several plan periods which presumes the presence of the same or similarly formulated priority in the plans for one, two or three periods.

Seventh - number of the priorities in plans In the Bulgarian practice of plan development, the matter of the number of priorities is especially up-to-date. Generally, the number of the priorities in the development plans should be limited to not more than three or four. The definition for priority itself suggests that the more priorities exist in certain plan, the more they lack sense because the sub-objectives will have the same statute of importance, i.e. when all sub-objectives in a plan are priorities, in fact there are no priorities. In the plans in 
which all or almost all sub-objectives are priorities, which sub-objective will be spared more resources? If all sub-objectives are priorities, this presumes relatively even distribution of the resources between the subobjectives, which is by itself in contradiction with the designation of the priority - to concentrate in absolute or relative sense more resource in the critical points for the system development.

\section{CONCLUSION}

The outlined seven divisions can serve as a benchmark and contribute to the improvement of the quality of plan documents in Bulgaria via the correct deducing of the priorities in them. This social economic state of the country, its territorial and economic units as well as citizens demonstrate that there is a pressing need for development and prosperity. The author satisfaction of this work would be to support this process.

\section{REFERENCES}

1. George F. Chadwick, A Systems View of Planning: Towards a Theory of the Urban and Regional Planning Process, 1975

2. Kaufman Roger, Oakley-Browne Hugh, Watkins Ryan, Leigh Doug, Strategic Planning for Success Aligning People, Performance, and Payoffs, 2003

3. Von Bertalanffy Ludwig, General System Theory - Foundations, Development, Applications, George Braziller, lnc.,1969

4. Saaty Thomas, The Analytic Hierarchy Process: Planning, Priority Setting, Resource Allocation, 1980 